\title{
Synthesizing Nanoencapsulated Sulfentrazone Herbicide and Optimizing Time and Dose for Season Long Weed Management in Irrigated Blackgram (Vigna mungo L.)
}

\author{
Vikram Kannamreddy, C.R. Chinnamuthu, S. Marimuthu', C. Bharathi
}

10.18805/LR-4447

\begin{abstract}
Background: Pulses are nutritious edible seeds of leguminous plants, have become an essential part of the human diet. Among the pulses, blackgram (Vigna mungo L.) is an important legume crop cultivated in tropical and subtropical regions of the world. Even though there are so many factors responsible for the lower yield of blackgram, weeds play a major role. Sulfentrazone is a broad spectrum herbicide belongs to the family of phenyl triazolinone. Sulfentrazone has high persistence and mobility with mean partition coefficient $\mathrm{K}_{\mathrm{oc}}=43$ and sorption coefficient $\mathrm{K}_{\mathrm{d}}<1$ and also has high horizontal and vertical leaching potential. Even though generally used herbicides in blackgram like pendimethalin, imazethapyr and quizalofop-ethyl are helpful in managing weeds, they have to apply multiple times or have to integrate with other methods of weed management which is expensive. So as to reduce the usage of multiple herbicides, to avoid manual weeding and to achieve season long weed control without affecting the environment the nano-encapsulated sulfentrazone is the better alternative and it gives better solution for the above constraints besides increasing the productivity.

Methods: Laboratory and field experiments were conducted in the Department of Agronomy, Tamil Nadu Agricultural University, Coimbatore during 2019-2020. Laboratory experiment was conducted to prepare nano-encapsulated sulfentrazone herbicide using solvent evaporation method. Screening trial was conducted in field with 17 treatments in randomized block design. Main trial was conducted with nine treatments of randomized block design by selecting the best performing treatments in screening trial. A confirmatory trial was also conducted by using same treatments.

Result: The encapsulated sulfentrazone particles were characterized in SEM (Scanning electron microscope) and also analysed with EDAX (Energy dispersive X-ray analysis) for elemental analysis, which is followed by particle size analysis and zeta potential to know the size and stability respectively. All these tests concluded that the sulfentrazone particles were encapsulated correctly and might be useful for slow release of the particle and also for reducing vertical and horizontal leachability. The field trials revealed that sulfentrazone @ $0.30 \mathrm{~kg}$ a.i. ha ${ }^{-1}$ with encapsulation applied at 1 DBS is better alternative for the season long weed management in blackgram without affecting the soil and ground water, as well as increasing the productivity.
\end{abstract}

Key words: Blackgram, Encapsulation, Solvent evaporation, Sulfentrazone, Weed.

\section{INTRODUCTION}

Pulses are nutritious edible seeds of leguminous plants, have become an essential part of the human diet. Among the pulses, blackgram (Vigna mungo L.) is an important legume crop cultivated in tropical and subtropical regions of the world. Even though there are so many factors responsible for the lower yield of blackgram weeds play a major role. Weeds are silent slayers of the crop because initially they grow along with crop, at certain stage they dominate crop and reduce the yield in a drastic way.

Sulfentrazone is a herbicidal molecule belongs to the family of phenyl triazolinone which controls the weeds by the process of protoporphyrinogen oxidase (PPO) inhibition. It can be applied as pre plant, pre-emergence or post-emergence for broad spectrum weed control (Dayan et al. 1996). Sulfentrazone has high persistence and mobility with mean partition coefficient $\mathrm{K}_{\mathrm{oc}}=43$ and sorption coefficient $\mathrm{K}_{\mathrm{d}}<1$ and also has high horizontal and vertical leaching potential (Martinez et al., 2008). It has high Groundwater Ubiquity Score (GUS) of 6.75 which is far more than broad spectrum
Department of Agronomy, Tamil Nadu Agricultural University, Coimbatore - 641 003, Tamil Nadu, India.

1Department of Nano Science and Technology, Tamil Nadu Agricultural University, Coimbatore -641 003, Tamil Nadu, India.

Corresponding Author: Vikram Kannamreddy, Department of Agronomy, Tamil Nadu Agricultural University, Coimbatore-641 003, Tamil Nadu, India. Email: vikramreddy2882@gmail.com

How to cite this article: Vikram, K.R., Chinnamuthu, C.R., Marimuthu, S. and Bharathi, C. (2021). Synthesizing Nanoencapsulated Sulfentrazone Herbicide and Optimizing Time and Dose for Season Long Weed Management in Irrigated Blackgram (Vigna mungo L.). Legume Research. DOI: 10.18805/ LR-4447.

Submitted: 22-06-2020 Accepted: 04-03-2021 Online: 22-04-2021

herbicides like pendimethalin and glyphosate which are having GUS of 0.66 and 0.42 respectively (Gustafson, 1989).

Even though generally used herbicides in blackgram like pendimethalin, imazethapyr and quizalofop-ethyl are 
helpful in managing weeds, they have to apply multiple times or have to integrate with other methods of weed management which is expensive. So as to reduce the usage of multiple herbicides, to avoid manual weeding and to achieve season long weed control without affecting the environment the nano-encapsulated sulfentrazone is the better alternative and it gives better solution for the above constraints besides increasing the productivity.

\section{MATERIALS AND METHODS \\ Preparation of encapsulated sulfentrazone}

One $\mathrm{g}$ of sulfentrazone (a.i) [i.e. $2.525 \mathrm{ml}$ of commercial formulation] was mixed with $10 \mathrm{ml}$ of ultrapure water and stirred it using magnetic stirrer for $5 \mathrm{~min}$. Then separately 2 $\mathrm{ml}$ of polyethylene glycol as polymer and $8 \mathrm{ml}$ of dichloromethane were taken, mixed and stirred for $5 \mathrm{~min}$ using magnetic stirrer. Both the solutions were mixed and stirred for another 5 min thus organic phase was formed. After that $4 \%$ starch solution was taken and stirred with magnetic stirrer for 1 hour thus formed the aqueous phase. Finally organic phase containing polymer with herbicide was added drop by drop to aqueous phase and stirred again with magnetic stirrer for 12 hours. Thus produced nanoparticles were collected as such (liquid formulation) in a vial or centrifuged it for $15 \mathrm{~min}$ at $5000 \mathrm{rpm}$ then dried the solid particles in vacuum desiccator to get dried powder.

All the field experiments were conducted at wetland farms, Tamil Nadu Agricultural University, Coimbatore during the year 2019-20 in randomized block design with three replications. The screening trial comprised of seventeen different treatments viz., sulfentrazone with and without encapsulation of two different concentrations @ 0.30 and $0.40 \mathrm{~kg}$ a.i. ha ${ }^{-1}$ and absolute control. The main field trial I and II comprised of nine treatments of which four best performed treatments were selected from the screening trial. Other treatments were pendimethalin @ $1.0 \mathrm{~kg}$ a.i. ha ${ }^{-1}$ at 2 DAS fb quizalofop-ethyl @ 50 g a.i. ha ${ }^{-1}$ and imazethapyr @
$50 \mathrm{~g}$ a.i. ha ${ }^{-1}$ at 20 DAS, pendimethalin @ $1.0 \mathrm{~kg}$ a.i. ha ${ }^{-1}$ at 2 DAS fb $1 \mathrm{HW}$ at 20 DAS, HW twice at 15 and 30 DAS, Weed free check and absolute control.

\section{RESULTS AND DISCUSSION}

\section{Lab experiment}

The encapsulated sulfentrazone particles were characterized in SEM (Fig 1) and also analysed with EDAX (Energy dispersive X-ray analysis) which is used for elemental analysis or chemical characterization of a sample. SEM images (Fig 1) were clearly showing that spherical or round particles which were encapsulated by the starch molecules. In the organic phase because of mixing and continuous stirring the polyethylene glycol polymerized and mixed with herbicide molecules in the presence of solvent dichloromethane. Similar results were observed by Mohanraj and Chen (2006). In the same way covalent bonding ability, mixing during preparation or surface adsorption ability of PEG was explained by Hans and Lowman (2002). Reis et al. (2006) explained in similar way about solvents used in organic phase as encapsulant and observed nano encapsulated particles were obtained by dispersion (Fig 1).

The SEM-EDAX image (Fig 2) is showing that presence of carbon and oxygen peak and also presence of little amounts of fluorine, Sulphur and chlorine confirmed the presence of active ingredient of sulfentrazone in the encapsulated herbicide. Dayan et al. (1998) given structure of sulfentrazone in the similar way which supports the above elemental composition. The spherical nanoparticles with smooth and shining surface is showing that herbicide was encapsulated with starch molecules (Fig 1).

In the particle size analyzer it was tested to know the size of encapsulated particles and zeta potential of the particles. The average particle size of encapsulated sulfentrazone and normal sulfentrazone were $186.9 \mathrm{~nm}$ (Fig 3) and $626.9 \mathrm{~nm}$ (Fig 4) respectively which were clearly

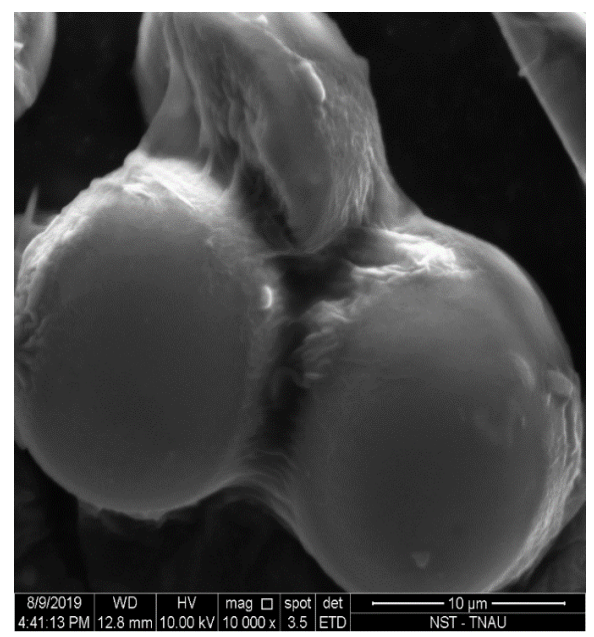

$\mathrm{B}$

Fig 1: SEM images at different magnifications of 1000x and 10000x ( $A$ and $B$ respectively). 
showing that encapsulated herbicidal particle size was far less than normal herbicide because of using solvent evaporation method by the processes of polymerization, dissociation and dispersion by the presence of polyethylene glycol, dichloromethane and starch. The zeta potential of encapsulated sulfentrazone was $-38.1 \mathrm{mV}$ (Fig 5). Zeta potential is a measure of surface charges present on the nano particles. Zeta potential shows the stability of the

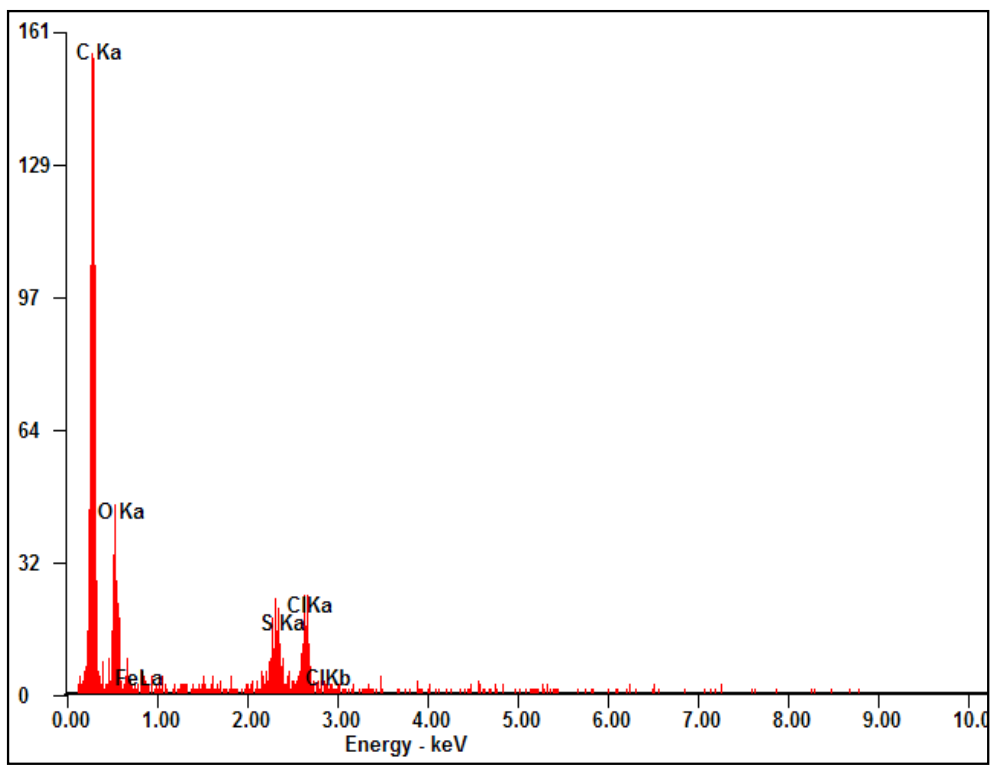

\begin{tabular}{||lll||}
\hline \hline Element & Wt\% & At\% \\
\hline $\boldsymbol{C K}$ & 66.06 & 78.26 \\
\hline $\boldsymbol{O K}$ & 16.32 & 14.51 \\
\hline $\boldsymbol{F K}$ & 01.21 & 00.31 \\
\hline $\boldsymbol{S K}$ & 07.80 & 03.46 \\
\hline $\boldsymbol{C I K}$ & 08.62 & 03.46 \\
\hline Matrix & Correction & ZAF \\
\hline \hline
\end{tabular}

Fig 2: SEM - EDAX values of encapsulated sulfentrazone herbicide.

\begin{tabular}{|c|c|c|c|c|}
\hline \multicolumn{5}{|c|}{ Measurement Results } \\
\hline \multirow{2}{*}{\multicolumn{4}{|c|}{ Date }} & : Tuesday, August 20, 2019 \\
\hline & nent Type & & & : Particle Size \\
\hline \multicolumn{4}{|c|}{ Sample Name } & : sulfendrazone 0.3 \\
\hline \multicolumn{4}{|c|}{ Scattering Angle } & 90 \\
\hline \multicolumn{4}{|c|}{ Temperature of the holder } & $25.1^{\circ} \mathrm{C}$ \\
\hline \multicolumn{4}{|c|}{ T\% before meas. } & $: 22170$ \\
\hline \multirow{2}{*}{\multicolumn{4}{|c|}{$\begin{array}{l}\text { Viscosity of the dispersion medium } \\
\text { Form Of Distribution }\end{array}$}} & : $0.893 \mathrm{mPa} \cdot \mathrm{s}$ \\
\hline & & & & Standard \\
\hline \multicolumn{4}{|c|}{ Representation of result } & : Scattering Light Intensity \\
\hline Count rá & & & & : 115 kCPS \\
\hline \multicolumn{5}{|c|}{ Calculation Results } \\
\hline Peak No. & S.P.Area Ratio & Mean & S.D. & Mode \\
\hline 1 & 1.00 & $190.1 \mathrm{~nm}$ & $14.3 \mathrm{~nm}$ & $186.9 \mathrm{~nm}$ \\
\hline 2 & $\cdots$ & & $\cdots \mathrm{nm}$ & $\cdots \mathrm{nm}$ \\
\hline 3 & $\ldots$ & $\cdots \mathrm{nm}$ & $\cdots \mathrm{nm}$ & $\cdots \mathrm{nm}$ \\
\hline Total & 1.00 & $190.1 \mathrm{~nm}$ & $14.3 \mathrm{~nm}$ & $186.9 \mathrm{~nm}$ \\
\hline
\end{tabular}

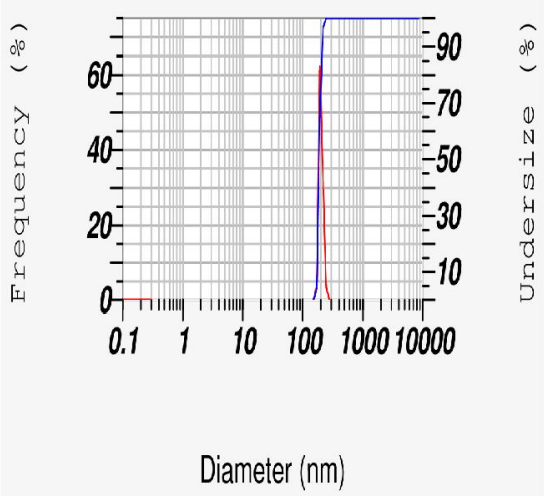

Fig 3: Particle size analyzer values for encapsulated sulfentrazone herbicide

Measurement Results

Date

Measurement Type

Sample Name

Scattering Angle

Temperature of the holder

T\% before meas.

Viscosity of the dispersion medium

Form Of Distribution

Representation of result

Count rate

Calculation Results

\begin{tabular}{|c|c|c|c|c|}
\hline Peak No. & S.P.Area Ratio & Mean & S. D. & Mode \\
\hline 1 & 1.00 & $649.8 \mathrm{~nm}$ & $93.5 \mathrm{~nm}$ & $626.9 \mathrm{~nm}$ \\
\hline 2 & $\cdots--$ & $\cdots-\cdots \mathrm{nm}$ & $\cdots-\cdots \mathrm{nm}$ & $\cdots-\cdots \mathrm{nm}$ \\
\hline 3 & $\cdots--$ & $\cdots-\mathrm{nm}$ & $\cdots-\cdots \mathrm{nm}$ & $\cdots-\mathrm{nm}$ \\
\hline Total & 1.00 & $649.8 \mathrm{~nm}$ & $93.5 \mathrm{~nm}$ & $626.9 \mathrm{~nm}$ \\
\hline
\end{tabular}

Tuesday, August 20, 2019

: Particle Size

: normal sulfendrazone

: 90

$: 25.1^{\circ} \mathrm{C}$

$: 31025$

: $0.893 \mathrm{mPa} \cdot \mathrm{s}$

: Standard

: Scattering Light Intensity

: 45 kCPS

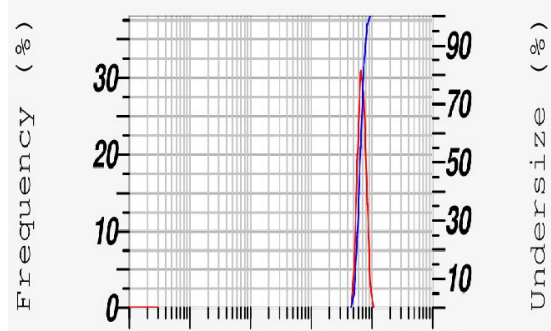

0.1110100100010000

Diameter (nm)

Fig 4: Particle size analyzer values of sulfentrazone (without encapsulation) herbicide 
colloids. Nano particles with zeta potential above (+/-) 30 $\mathrm{mV}$ have been considered to be stable in suspension, as the charge on the surface of particles prevents aggregation. As the zeta potential of encapsulated sulfentrazone was $38.1 \mathrm{mV}$ which is more than (+/-) $30 \mathrm{mV}$, thus the encapsulated sulfentrazone herbicide is stable. This stability might be useful for slow release of the particle and also for reducing vertical and horizontal leachability.

\section{Field experiment}

Weed flora

Weed species vegetation in the screening trial consists of Dinebra retroflexa, Echinochloa colonum, Amaranthus viridis, Calotropis gigantea, Corchorus trilocularis, Euphorbia thymifolia, Malachra capitata, Parthenium hysterophorus, Portulaca oleracea, Trianthema portulacastrum. All these weeds were occurred in main field trial I and II also except Calotropis gigantea and Parthenium hysterophorus. Among these most dominant weed species were grasses. Similar findings were observed by Dayan et al. (1996).

\section{Screening trial}

\section{Effect of weed management treatments on weeds}

The higher weed density was observed in unweeded control respectively at 20, 40 and 60 DAS. Lower weed density was noticed in the treatments $T_{7}$ (Sulfentrazone @ $0.40 \mathrm{~kg}$ a.i. $\mathrm{ha}^{-1} \mathrm{e}^{+}$at 2 DAS) and $\mathrm{T}_{8}$ (Sulfentrazone @ $0.40 \mathrm{~kg}$ a.i. ha ${ }^{-1}$ $\mathrm{e}^{+}$at 3 DAS) at 20 and 40 DAS respectively. But at 60 DAS
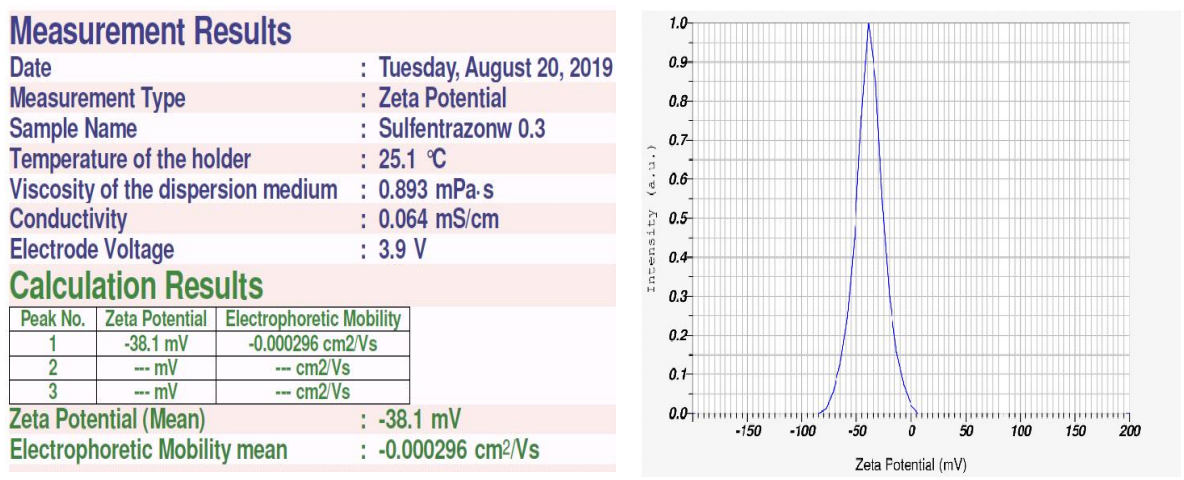

Fig 5: Zeta potential values for encapsulated sulfentrazone herbicide.

Table 1. Effect of weed management treatments on total weed density, Seed, Hull and Haulm yield of blackgram in screening trial.

\begin{tabular}{|c|c|c|c|c|c|c|c|}
\hline \multirow{2}{*}{ T. No } & \multirow{2}{*}{ Treatments } & \multicolumn{3}{|c|}{ Total weed density (No. m²) } & \multirow{2}{*}{$\begin{array}{c}\text { Seed } \\
\text { yield } \\
\left(\mathrm{kg} \mathrm{ha}^{-1}\right)\end{array}$} & \multirow{2}{*}{$\begin{array}{c}\text { Hull } \\
\text { yield } \\
\left(\mathrm{kg} \mathrm{ha}^{-1}\right)\end{array}$} & \multirow{2}{*}{$\begin{array}{c}\text { Haulm } \\
\text { yield } \\
\text { (kg ha }^{-1}\end{array}$} \\
\hline & & 20 DAS & 40 DAS & 60 DAS & & & \\
\hline $\mathrm{T}_{1}$ & Sulfentrazone @ $0.30 \mathrm{~kg}$ a.i. ha ${ }^{-1} \mathrm{e}^{+}$at 1 DBS & $1.46(1.67)$ & $1.56(2.00)$ & $1.86(3.00)$ & 1349 & 587 & 3851 \\
\hline $\mathrm{T}_{2}$ & Sulfentrazone @ $0.30 \mathrm{~kg}$ a.i. ha ${ }^{-1} \mathrm{e}^{+}$at $1 \mathrm{DAS}$ & $4.74(22.00)$ & $5.37(28.33)$ & $6.12(37.00)$ & 809 & 352 & 2337 \\
\hline $\mathrm{T}_{3}$ & Sulfentrazone @ $0.30 \mathrm{~kg}$ a.i. ha ${ }^{-1} \mathrm{e}^{+}$at 2 DAS & $1.68(2.33)$ & $1.86(3.00)$ & $2.54(6.00)$ & 1276 & 555 & 3646 \\
\hline $\mathrm{T}_{4}$ & Sulfentrazone @ $0.30 \mathrm{~kg}$ a.i. ha $\mathrm{a}^{-1} \mathrm{e}^{+}$at 3 DAS & $1.56(2.00)$ & $1.77(2.67)$ & $2.65(6.67)$ & 1062 & 462 & 3366 \\
\hline $\mathrm{T}_{5}$ & Sulfentrazone @ $0.40 \mathrm{~kg}$ a.i. ha ${ }^{-1} \mathrm{e}^{+}$at 1 DBS & $1.34(1.33)$ & $1.46(1.67)$ & $1.77(2.67)$ & 1355 & 589 & 3842 \\
\hline $\mathrm{T}_{6}$ & Sulfentrazone @ $0.40 \mathrm{~kg}$ a.i.ha $\mathrm{e}^{-1} \mathrm{e}^{+}$at $1 \mathrm{DAS}$ & $4.41(19.00)$ & $5.01(24.67)$ & $5.76(32.67)$ & 783 & 341 & 2292 \\
\hline $\mathrm{T}_{7}$ & Sulfentrazone @ $0.40 \mathrm{~kg}$ a.i. ha ${ }^{-1} \mathrm{e}^{+}$at 2 DAS & $0.71(0.00)$ & $0.88(0.33)$ & $2.19(4.33)$ & 1095 & 476 & 3238 \\
\hline $\mathrm{T}_{8}$ & Sulfentrazone @ $0.40 \mathrm{~kg}$ a.i. ha ${ }^{-1} \mathrm{e}^{+}$at 3 DAS & $0.71(0.00)$ & $0.88(0.33)$ & $2.34(5.00)$ & 1099 & 478 & 3250 \\
\hline$T_{9}$ & Sulfentrazone @ $0.30 \mathrm{~kg}$ a.i. ha ${ }^{-1} \mathrm{e}^{-}$at 1 DBS & $2.04(3.67)$ & $2.41(5.33)$ & $3.31(10.67)$ & 1249 & 543 & 3562 \\
\hline $\mathrm{T}_{10}$ & Sulfentrazone @ $0.30 \mathrm{~kg}$ a.i. ha ${ }^{-1} \mathrm{e}^{-}$at 1 DAS & $5.18(26.33)$ & $5.84(33.67)$ & $6.74(45.00)$ & 761 & 331 & 2238 \\
\hline$T_{11}$ & Sulfentrazone @ $0.30 \mathrm{~kg}$ a.i. ha ${ }^{-1} \mathrm{e}^{-}$at 2 DAS & $2.34(5.00)$ & $2.48(5.67)$ & $3.17(9.67)$ & 1182 & 514 & 3338 \\
\hline $\mathrm{T}_{12}$ & Sulfentrazone @ $0.30 \mathrm{~kg}$ a.i. ha ${ }^{-1} \mathrm{e}^{-}$at 3 DAS & $2.18(4.33)$ & $2.41(5.33)$ & $3.26(10.33)$ & 945 & 411 & 2734 \\
\hline $\mathrm{T}_{13}$ & Sulfentrazone @ $0.40 \mathrm{~kg}$ a.i. ha ${ }^{-1} \mathrm{e}^{-}$at 1 DBS & $1.66(2.33)$ & $1.93(3.33)$ & $3.02(8.67)$ & 1184 & 515 & 3353 \\
\hline $\mathrm{T}_{14}$ & Sulfentrazone @ $0.40 \mathrm{~kg}$ a.i. ha ${ }^{-1} \mathrm{e}^{-}$at 1 DAS & $4.84(23.00)$ & $5.52(30.00)$ & $6.41(40.67)$ & 706 & 307 & 2061 \\
\hline $\mathrm{T}_{15}$ & Sulfentrazone @ $0.40 \mathrm{~kg}$ a.i. ha ${ }^{-1} \mathrm{e}^{-}$at 2 DAS & $2.04(3.67)$ & $2.20(4.33)$ & $2.95(8.33)$ & 795 & 346 & 2309 \\
\hline $\mathrm{T}_{16}$ & Sulfentrazone @ $0.40 \mathrm{~kg}$ a.i. ha ${ }^{-1} \mathrm{e}^{-}$at 3 DAS & $2.11(4.00)$ & $2.20(4.33)$ & $3.02(8.67)$ & 825 & 359 & 2422 \\
\hline \multirow[t]{3}{*}{$\mathrm{T}_{17}$} & Absolute control & $9.19 \quad(84.00)$ & $10.17(103.00)$ & $11.08(122.33)$ & 328 & 142 & 1434 \\
\hline & SEd & 0.20 & 0.17 & 0.22 & 49 & 21 & 191 \\
\hline & $C D(P=0.05)$ & 0.40 & 0.35 & 0.45 & 100 & 44 & 389 \\
\hline
\end{tabular}

Data subjected to square root [" $(\mathrm{X}+0.5)$ ] transformation. Values in parenthesis are means of original values $\mathrm{e}^{+}$- with encapsulation $\quad \mathrm{e}^{-}$- without encapsulation $\quad$ DBS - Day before sowing DAS - Day(s) after sowing. 
lower weed density was noticed in $T_{1}$ (Sulfentrazone @ 0.30 $\mathrm{kg}$ a.i.ha $\mathrm{e}^{-1} \mathrm{e}^{+}$at $1 \mathrm{DBS}$ ) and $\mathrm{T}_{5}$ (Sulfentrazone @ $0.40 \mathrm{~kg}$ a.i. ha $^{-1} \mathrm{e}^{+}$at 1 DBS) (Table 1 ). The results were in conformity with the findings of Srivastava (2003). He proved that in hand weeded plot weed density was $43.53 \mathrm{~m}^{-2}$ whereas in the sulfentrazone $\left(0.8 \mathrm{~L} \mathrm{ha}^{-1}\right)$ treated plot the weed density was only $0.90 \mathrm{~m}^{-2}$.

Effect of weed management treatments on blackgram crop

Plant height $(\mathrm{cm})$ was lesser in all the sulfentrazone applied treatments compared to absolute control (15.87 and 49.73)

Table 2: Effect of weed management treatments on plant height and plant dry weight of blackgram in screening trial.

\begin{tabular}{|c|c|c|c|c|c|c|c|}
\hline \multirow{2}{*}{ T. No } & \multirow{2}{*}{ Treatments } & \multicolumn{3}{|c|}{ Plant height $(\mathrm{cm})$} & \multicolumn{3}{|c|}{ Plant dry weight (g plant ${ }^{-1}$ ) } \\
\hline & & 20 DAS & 40 DAS & 60 DAS & 20 DAS & 40 DAS & 60 DAS \\
\hline $\mathrm{T}_{1}$ & Sulfentrazone @ $0.30 \mathrm{~kg}$ a.i. ha $\mathrm{h}^{-1} \mathrm{e}^{+}$at 1 DBS & 13.27 & 43.60 & 54.27 & 1.88 & 7.73 & 16.83 \\
\hline $\mathrm{T}_{2}$ & Sulfentrazone $@ 0.30 \mathrm{~kg}$ a.i. ha $\mathrm{e}^{-1} \mathrm{e}^{+}$at 1 DAS & 13.77 & 42.70 & 49.73 & 1.76 & 4.26 & 10.35 \\
\hline $\mathrm{T}_{3}$ & Sulfentrazone @ $0.30 \mathrm{~kg}$ a.i. ha $\mathrm{h}^{-1} \mathrm{e}^{+}$at 2 DAS & 13.43 & 43.77 & 54.73 & 1.87 & 7.22 & 15.95 \\
\hline $\mathrm{T}_{4}$ & Sulfentrazone @ $0.30 \mathrm{~kg}$ a.i. ha $\mathrm{h}^{-1} \mathrm{e}^{+}$at 3 DAS & 12.93 & 40.83 & 50.57 & 1.78 & 6.92 & 14.73 \\
\hline $\mathrm{T}_{5}$ & Sulfentrazone @ $0.40 \mathrm{~kg}$ a.i. ha $\mathrm{e}^{-1} \mathrm{e}^{+}$at 1 DBS & 13.13 & 43.03 & 53.50 & 1.86 & 7.71 & 16.90 \\
\hline $\mathrm{T}_{6}$ & Sulfentrazone @ $0.40 \mathrm{~kg}$ a.i. ha $\mathrm{h}^{-1} \mathrm{e}^{+}$at $1 \mathrm{DAS}$ & 13.53 & 42.47 & 49.47 & 1.75 & 4.14 & 10.04 \\
\hline $\mathrm{T}_{7}$ & Sulfentrazone @ $0.40 \mathrm{~kg}$ a.i. ha ${ }^{-1} \mathrm{e}^{+}$at 2 DAS & 12.87 & 38.83 & 48.50 & 1.65 & 5.82 & 14.11 \\
\hline $\mathrm{T}_{8}$ & Sulfentrazone @ $0.40 \mathrm{~kg}$ a.i. ha $\mathrm{h}^{-1} \mathrm{e}^{+}$at 3 DAS & 12.83 & 38.53 & 47.40 & 1.65 & 6.04 & 14.19 \\
\hline $\mathrm{T}_{9}$ & Sulfentrazone @ $0.30 \mathrm{~kg}$ a.i. ha ${ }^{-1} \mathrm{e}^{-}$at 1 DBS & 13.10 & 41.10 & 53.63 & 1.84 & 7.46 & 15.63 \\
\hline $\mathrm{T}_{10}$ & Sulfentrazone @ $0.30 \mathrm{~kg}$ a.i. ha ${ }^{-1} \mathrm{e}^{-}$at 1 DAS & 13.70 & 41.83 & 47.87 & 1.75 & 4.17 & 9.77 \\
\hline$T_{11}$ & Sulfentrazone @ $0.30 \mathrm{~kg}$ a.i. ha ${ }^{-1} \mathrm{e}^{-}$at 2 DAS & 13.30 & 42.03 & 53.50 & 1.83 & 7.16 & 14.83 \\
\hline $\mathrm{T}_{12}$ & Sulfentrazone @ $0.30 \mathrm{~kg}$ a.i. ha ${ }^{-1} \mathrm{e}^{-}$at 3 DAS & 12.33 & 37.83 & 42.83 & 1.61 & 6.03 & 11.98 \\
\hline $\mathrm{T}_{13}$ & Sulfentrazone @ $0.40 \mathrm{~kg}$ a.i. ha ${ }^{-1} \mathrm{e}^{-}$at 1 DBS & 12.77 & 41.57 & 49.63 & 1.83 & 7.10 & 14.84 \\
\hline $\mathrm{T}_{14}$ & Sulfentrazone @ $0.40 \mathrm{~kg}$ a.i. ha-1 $\mathrm{e}^{-}$at 1 DAS & 13.20 & 37.17 & 42.53 & 1.75 & 4.00 & 9.11 \\
\hline $\mathrm{T}_{15}$ & Sulfentrazone @ $0.40 \mathrm{~kg}$ a.i. ha ${ }^{-1} \mathrm{e}^{-}$at 2 DAS & 12.13 & 30.37 & 38.27 & 1.58 & 6.16 & 10.18 \\
\hline $\mathrm{T}_{16}$ & Sulfentrazone @ $0.40 \mathrm{~kg}$ a.i. ha ${ }^{-1} \mathrm{e}^{-}$at 3 DAS & 12.07 & 31.90 & 40.53 & 1.56 & 5.39 & 10.54 \\
\hline \multirow[t]{3}{*}{$T_{17}$} & Absolute control & 15.87 & 49.73 & 55.07 & 1.79 & 3.44 & 6.23 \\
\hline & SEd & 0.70 & 2.28 & 2.56 & 0.18 & 0.40 & 0.72 \\
\hline & $\mathrm{CD}(\mathrm{P}=0.05)$ & 1.42 & 4.64 & 5.22 & NS & 0.81 & 1.47 \\
\hline
\end{tabular}

Table 3. Effect of weed management treatments on density of total weeds (No. $\mathrm{m}^{-2}$ ) in main trial I and II.

\begin{tabular}{|c|c|c|c|c|c|c|c|}
\hline \multirow{2}{*}{ T. No. } & \multirow{2}{*}{ Treatments } & \multicolumn{3}{|c|}{ Main trial I } & \multicolumn{3}{|c|}{ Main trial II } \\
\hline & & 20DAS & 40 DAS & 60 DAS & 20 DAS & 40 DAS & 60 DAS \\
\hline \multirow[t]{2}{*}{$\mathrm{T}_{1}$} & Sulfentrazone @ $0.3 \mathrm{~kg}$ a.i. ha ${ }^{-1} \mathrm{e}^{+}$at 1 DBS & 0.71 & 1.39 & 2.03 & 0.71 & 1.47 & 1.77 \\
\hline & & $(0.00)$ & $(1.67)$ & $(3.67)$ & $(0.00)$ & $(2.00)$ & $(2.67)$ \\
\hline \multirow[t]{2}{*}{$\mathrm{T}_{2}$} & Sulfentrazone @ $0.3 \mathrm{~kg}$ a.i. ha ${ }^{-1} \mathrm{e}^{-}$at 1 DBS & 1.10 & 1.93 & 2.66 & 1.00 & 2.02 & 2.32 \\
\hline & & $(1.00)$ & (3.33) & $(6.67)$ & $(0.67)$ & $(3.67)$ & $(5.00)$ \\
\hline \multirow[t]{2}{*}{$\mathrm{T}_{3}$} & Sulfentrazone @ $0.3 \mathrm{~kg}$ a.i. ha ${ }^{-1} \mathrm{e}^{+}$at 2 DAS & 2.60 & 2.74 & 3.76 & 2.83 & 3.19 & 3.74 \\
\hline & & $(6.33)$ & $(7.33)$ & $(13.67)$ & $(7.67)$ & $(9.67)$ & $(13.67)$ \\
\hline \multirow[t]{2}{*}{$\mathrm{T}_{4}$} & Sulfentrazone @ $0.3 \mathrm{~kg}$ a.i. ha ${ }^{-1} \mathrm{e}^{-}$at 2 DAS & 2.73 & 2.91 & 4.22 & 3.00 & 3.69 & 4.22 \\
\hline & & $(7.00)$ & $(8.00)$ & $(17.33)$ & $(8.67)$ & $(13.33)$ & $(17.33)$ \\
\hline \multirow[t]{2}{*}{$\mathrm{T}_{5}$} & Pendimethalin @1.0 kg a.i. ha ${ }^{-1}$ at 2 DAS fb Quizalofop-ethyl & 4.13 & 2.73 & 4.29 & 5.04 & 3.02 & 4.14 \\
\hline & @ $50 \mathrm{~g}$ a.i. ha ${ }^{-1}$ and Imazethapyr @ $50 \mathrm{~g}$ a.i. ha ${ }^{-1}$ at 20 DAS & $(16.67)$ & $(16.67)$ & $(7.00)$ & $(18.00)$ & $(25.00)$ & $(8.67)$ \\
\hline \multirow[t]{2}{*}{$\mathrm{T}_{6}$} & Pendimethalin @1.0 kg a.i. ha ${ }^{-1}$ at 2 DAS $f b 1 \mathrm{HW}$ at 20 DAS & 4.11 & 3.39 & 3.71 & 4.90 & 3.62 & 3.87 \\
\hline & & $(16.67)$ & $(11.00)$ & $(13.33)$ & (23.67) & $(12.67)$ & $(14.67)$ \\
\hline \multirow[t]{2}{*}{$\mathrm{T}_{7}$} & HW twice at 15 and 30 DAS & 0.71 & 1.77 & 3.56 & 0.71 & 1.93 & 3.62 \\
\hline & & $(0.00)$ & $(2.67)$ & $(12.33)$ & $(0.00)$ & $(3.33)$ & $(12.67)$ \\
\hline \multirow[t]{2}{*}{$\mathrm{T}_{8}$} & Weed free check & 0.71 & 0.71 & 0.71 & 0.71 & 0.71 & 0.71 \\
\hline & & $(0.00)$ & $(0.00)$ & $(0.00)$ & $(0.00)$ & $(0.00)$ & $(0.00)$ \\
\hline \multirow[t]{4}{*}{$T_{9}$} & Absolute control & 9.99 & 10.67 & 11.68 & 11.70 & 12.38 & 12.65 \\
\hline & & (99.33) & (113.33) & $(136.00)$ & $(136.67)$ & $(153.00)$ & (159.67) \\
\hline & SEd & 0.29 & 0.28 & 0.27 & 0.37 & 0.33 & 0.27 \\
\hline & $C D(P=0.05)$ & 0.62 & 0.59 & 0.56 & 0.78 & 0.70 & 0.57 \\
\hline
\end{tabular}

Data subjected to square root [" $(\mathrm{X}+0.5)]$ transformation. Values in parenthesis are means of original values. 
at 20 and 40 DAS. But at 60 DAS the plant height was on par in $\mathrm{T}_{1} \mathrm{~T}_{3} \mathrm{~T}_{4} \mathrm{~T}_{5}, \mathrm{~T}_{9}$ and $\mathrm{T}_{11}$ with the control (55.07) (Table 2). In case of plant dry weight $\left(\mathrm{g} \mathrm{plant}^{-1}\right)$ at 20 DAS there was no significant difference among the treatments. At 40 and 60 DAS more dry weight was noticed in $T_{1}, T_{3}, T_{5}, T_{9}$ and $T_{11}$ compared to unweeded control (Table 2).

Seed yield, hull yield and haulm yield $\left(\mathrm{kg} \mathrm{ha}^{-1}\right)$ of blackgram were higher in sulfentrazone @ $0.30\left(\mathrm{~T}_{1}\right)$ and $0.40\left(\mathrm{~T}_{5}\right) \mathrm{kg}$ a.i. ha $^{-1}$ at 1 DBS which are 1349, 587 and $3851 \mathrm{~kg} \mathrm{ha}^{-1}$ in $\mathrm{T}_{1}$ and 1355, 589 and $3842 \mathrm{~kg} \mathrm{ha}^{-1}$, respectively (Table 1). Similar results were recorded by Krausz et al. (1998) in soybean when sulfentrazone applied @ 0.42 kg a.i. ha ${ }^{-1}$.

\section{Main trial I and II}

\section{Effect of weed management treatments on weeds}

Zero weed density was noticed with the application of encapsulated sulfentrazone at $1 \mathrm{DBS}\left(\mathrm{T}_{1}\right)$, hand weeding at
15 and 30 DAS $\left(T_{7}\right)$ and weed free plot $\left(T_{8}\right)$ in both the trials at 20 DAS. Very low weed density was observed in $T_{1}$ and $\mathrm{T}_{2}$ (sulfentrazone without encapsulation applied at 1 DBS) and $T_{7}$ in both the trials at 40 and 60 DAS. All the other treatments also noticed lower weed population compared to absolute control in both main trials (Table 3 ). The results were in conformity with the findings of Srivastava (2003). In case of pendimethalin similar weed density was observed by Gupta et al. (2013).

\section{Effect of weed management treatments on blackgram crop}

In plant dry matter at 20 DAS there was no significant difference among the treatments. At 40 and 60 DAS more plant dry weight was noticed in all the treatments compared to control (Table 4). Seed yield, hull yield and haulm yield of blackgram were higher in $\mathrm{T}_{1}, \mathrm{~T}_{2}, \mathrm{~T}_{5}, \mathrm{~T}_{7}$ and $\mathrm{T}_{8}$ in both trials (Table 5). There was no significant difference in harvest

Table 4. Effect of weed management treatments on dry matter production (g plant ${ }^{-1}$ ) of blackgram in main trial I and II.

\begin{tabular}{|c|c|c|c|c|c|c|c|}
\hline \multirow{3}{*}{ T. No. } & \multirow{3}{*}{ Treatments } & \multicolumn{3}{|c|}{ Main trial I } & \multicolumn{3}{|c|}{ Main trial II } \\
\hline & & 20 & 40 & 60 & 20 & 40 & 60 \\
\hline & & DAS & DAS & DAS & DAS & DAS & DAS \\
\hline $\mathrm{T}_{1}$ & Sulfentrazone @ $0.3 \mathrm{~kg}$ a.i. ha ${ }^{-1} \mathrm{e}^{+}$at 1 DBS & 1.73 & 6.43 & 14.63 & 1.79 & 6.67 & 15.43 \\
\hline $\mathrm{T}_{2}$ & Sulfentrazone @ $0.3 \mathrm{~kg}$ a.i. ha ${ }^{-1} \mathrm{e}^{-}$at 1 DBS & 1.73 & 6.37 & 14.50 & 1.77 & 6.61 & 15.34 \\
\hline $\mathrm{T}_{3}$ & Sulfentrazone @ $0.3 \mathrm{~kg}$ a.i. ha ${ }^{-1} \mathrm{e}^{+}$at 2 DAS & 1.69 & 6.29 & 14.33 & 1.72 & 6.53 & 15.18 \\
\hline $\mathrm{T}_{4}$ & Sulfentrazone @ 0.3 kg a.i. ha ${ }^{-1} \mathrm{e}^{-}$at 2 DAS & 1.68 & 6.24 & 14.07 & 1.69 & 6.48 & 14.98 \\
\hline $\mathrm{T}_{5}$ & $\begin{array}{l}\text { Pendimethalin @ } 1.0 \mathrm{~kg} \text { a.i. ha- }{ }^{-1} \text { at } 2 \text { DAS fb Quizalofop-ethyl } \\
\text { @ } 50 \mathrm{~g} \text { a.i. ha }{ }^{-1} \text { and Imazethapyr @ } 50 \mathrm{~g} \text { a.i. ha }{ }^{-1} \text { at } 20 \text { DAS }\end{array}$ & 1.72 & 6.38 & 14.41 & 1.77 & 6.63 & 15.27 \\
\hline $\mathrm{T}_{6}$ & Pendimethalin @1.0 kg a.i. ha ${ }^{-1}$ at 2 DAS $f b 1 \mathrm{HW}$ at 20 DAS & 1.73 & 6.35 & 14.37 & 1.76 & 6.59 & 15.24 \\
\hline $\mathrm{T}_{7}$ & $\mathrm{HW}$ twice at 15 and $30 \mathrm{DAS}$ & 1.75 & 6.45 & 14.76 & 1.78 & 6.70 & 15.60 \\
\hline $\mathrm{T}_{8}$ & Weed free check & 1.77 & 6.51 & 14.84 & 1.82 & 6.76 & 15.73 \\
\hline \multirow[t]{3}{*}{$\mathrm{T}_{9}$} & Absolute control & 1.71 & 2.69 & 5.41 & 1.74 & 2.80 & 5.77 \\
\hline & SEd & 0.16 & 0.30 & 0.82 & 0.20 & 0.31 & 0.74 \\
\hline & $\mathrm{CD}(\mathrm{P}=0.05)$ & NS & 0.64 & 1.74 & NS & 0.66 & 1.57 \\
\hline
\end{tabular}

Table 5. Effect of weed management treatments on seed yield $\left(\mathrm{kg} \mathrm{ha}^{-1}\right)$, hull or bhusa yield $\left(\mathrm{kg} \mathrm{ha}^{-1}\right)$, haulm yield $\left(\mathrm{kg} \mathrm{ha}^{-1}\right)$ and harvest index of blackgram in main trial I and II.

\begin{tabular}{|c|c|c|c|c|c|c|c|c|c|}
\hline \multirow[b]{2}{*}{ T. No. } & \multirow[b]{2}{*}{ Treatments } & \multicolumn{4}{|c|}{ Main trial I } & \multicolumn{4}{|c|}{ Main trial II } \\
\hline & & $\begin{array}{l}\text { Seed } \\
\text { yield }\end{array}$ & $\begin{array}{l}\text { Hull } \\
\text { yield }\end{array}$ & $\begin{array}{r}\text { Haulm } \\
\text { yield }\end{array}$ & $\begin{array}{l}\text { Harvest } \\
\text { index }\end{array}$ & $\begin{array}{l}\text { Seed } \\
\text { yield }\end{array}$ & $\begin{array}{l}\text { Hull } \\
\text { yield }\end{array}$ & $\begin{array}{l}\text { Haulm } \\
\text { yield }\end{array}$ & $\begin{array}{r}\text { Harvest } \\
\text { index }\end{array}$ \\
\hline $\mathrm{T}_{1}$ & Sulfentrazone @ $0.3 \mathrm{~kg}$ a.i. ha ${ }^{-1} \mathrm{e}^{+}$at 1 DBS & 1229 & 539 & 3588 & 0.23 & 1303 & 564 & 3687 & 0.23 \\
\hline $\mathrm{T}_{2}$ & Sulfentrazone @ $0.3 \mathrm{~kg}$ a.i. ha ${ }^{-1} \mathrm{e}^{-}$at 1 DBS & 1214 & 533 & 3555 & 0.23 & 1272 & 551 & 3666 & 0.23 \\
\hline $\mathrm{T}_{3}$ & Sulfentrazone @ $0.3 \mathrm{~kg}$ a.i. ha ${ }^{-1} \mathrm{e}^{+}$at 2 DAS & 1123 & 493 & 3514 & 0.22 & 1177 & 510 & 3628 & 0.22 \\
\hline $\mathrm{T}_{4}$ & Sulfentrazone @ $0.3 \mathrm{~kg}$ a.i. ha ${ }^{-1} \mathrm{e}^{-}$at 2 DAS & 1095 & 480 & 3451 & 0.22 & 1134 & 491 & 3578 & 0.22 \\
\hline \multirow[t]{2}{*}{$\mathrm{T}_{5}$} & $\begin{array}{l}\text { Pendimethalin @ } 1.0 \mathrm{~kg} \text { a.i. ha }{ }^{-1} \text { at } 2 \text { DAS } \\
\text { fb Quizalofop-ethyl @ } 50 \mathrm{~g} \text { a.i. ha and }\end{array}$ & & & & & & & & \\
\hline & Imazethapyr @ 50 g a.i. ha-1 at 20 DAS & 1218 & 534 & 3534 & 0.23 & 1231 & 533 & 3649 & 0.23 \\
\hline $\mathrm{T}_{6}$ & $\begin{array}{l}\text { Pendimethalin @ } 1.0 \mathrm{~kg} \text { a.i. ha }{ }^{-1} \text { at } 2 \text { DAS } \\
\text { fb } 1 \mathrm{HW} \text { at } 20 \mathrm{DAS}\end{array}$ & 1150 & 505 & 3524 & 0.22 & 1192 & 516 & 3640 & 0.22 \\
\hline $\mathrm{T}_{7}$ & $\mathrm{HW}$ twice at 15 and $30 \mathrm{DAS}$ & 1282 & 563 & 3620 & 0.24 & 1327 & 575 & 3726 & 0.24 \\
\hline $\mathrm{T}_{8}$ & Weed free check & 1301 & 571 & 3640 & 0.24 & 1388 & 601 & 3758 & 0.24 \\
\hline \multirow[t]{3}{*}{$\mathrm{T}_{9}$} & Absolute control & 381 & 167 & 1362 & 0.20 & 374 & 162 & 1375 & 0.20 \\
\hline & SEd & 120 & 53 & 201 & 0.02 & 123 & 53 & 172 & 0.02 \\
\hline & $\mathrm{CD}(\mathrm{P}=0.05)$ & 255 & 112 & 427 & NS & 261 & 113 & 365 & NS \\
\hline
\end{tabular}


Synthesizing Nanoencapsulated Sulfentrazone Herbicide and Optimizing Time and Dose for Season Long Weed Management in ...

Table 6. Effect of weed management treatments on economics of blackgram in main trial I and II

\begin{tabular}{|c|c|c|c|c|c|}
\hline \multirow[b]{2}{*}{ T. No. } & \multirow[b]{2}{*}{ Treatments } & \multicolumn{4}{|c|}{ Main trial I and $\mathrm{II}^{*}$} \\
\hline & & $\begin{array}{l}\text { Cost of cultivation } \\
\qquad\left(\mathrm{Rs} \mathrm{ha}^{-1}\right)\end{array}$ & $\begin{array}{l}\text { Gross return } \\
\left(\text { Rs ha } \text { ha }^{-1}\right)\end{array}$ & $\begin{array}{l}\text { Net returns } \\
\left(\text { Rs ha }^{-1}\right)\end{array}$ & $\mathrm{B}: \mathrm{C}$ ratio \\
\hline $\mathrm{T}_{1}$ & Sulfentrazone @ $0.3 \mathrm{~kg}$ a.i. ha ${ }^{-1} \mathrm{e}^{+}$at 1 DBS & 27685 & 92233 & 64548 & 3.33 \\
\hline $\mathrm{T}_{2}$ & Sulfentrazone @ $0.3 \mathrm{~kg}$ a.i. ha ${ }^{-1} \mathrm{e}^{-}$at 1 DBS & 27135 & 90063 & 62928 & 3.32 \\
\hline $\mathrm{T}_{3}$ & Sulfentrazone @ $0.3 \mathrm{~kg}$ a.i. ha ${ }^{-1} \mathrm{e}^{+}$at 2 DAS & 27685 & 84064 & 56379 & 3.04 \\
\hline $\mathrm{T}_{4}$ & Sulfentrazone @ $0.3 \mathrm{~kg}$ a.i. ha ${ }^{-1} \mathrm{e}^{-}$at 2 DAS & 27135 & 81513 & 54378 & 3.00 \\
\hline \multirow[t]{2}{*}{$\mathrm{T}_{5}$} & $\begin{array}{l}\text { Pendimethalin @ } 1.0 \mathrm{~kg} \text { a.i. ha- }{ }^{-1} \text { at } 2 \text { DAS fb } \\
\text { Quizalofop-ethyl @ } 50 \mathrm{~g} \text { a.i. ha }{ }^{-1} \text { and }\end{array}$ & & & & \\
\hline & Imazethapyr @ 50 g a.i. ha-1 at 20 DAS & 28385 & 89314 & 60929 & 3.15 \\
\hline $\mathrm{T}_{6}$ & $\begin{array}{l}\text { Pendimethalin @ } 1.0 \mathrm{~kg} \text { a.i. ha }{ }^{-1} \text { at } 2 \text { DAS } \\
\mathrm{fb} 1 \mathrm{HW} \text { at } 20 \mathrm{DAS}\end{array}$ & 31925 & 85535 & 53610 & 2.68 \\
\hline $\mathrm{T}_{7}$ & $\mathrm{HW}$ twice at 15 and $30 \mathrm{DAS}$ & 33895 & 95005 & 61110 & 2.80 \\
\hline $\mathrm{T}_{8}$ & Weed free check & 36175 & 97812 & 61637 & 2.70 \\
\hline $\mathrm{T}_{9}$ & Absolute control & 24775 & 27808 & 3033 & 1.12 \\
\hline
\end{tabular}

* Average values of main trial I and II were taken for calculating economics

Data not statistically analysed.

index of all the treatments (Table 5). Higher gross returns $\left(92,233 \mathrm{Rs} \mathrm{ha}^{-1}\right)$, net returns $\left(64,548 \mathrm{Rs} \mathrm{ha}^{-1}\right)$ and $\mathrm{B}: \mathrm{C}$ ratio (3.33) were acquired in $T_{1}$ followed by $T_{2}$ and least Gross returns $\left(27,808 \mathrm{Rs} \mathrm{ha}^{-1}\right)$ and net returns $\left(3,033 \mathrm{Rs}^{\mathrm{h}} \mathrm{h}^{-1}\right)$ and $B: C$ ratio (1.12) were acquired in absolute control (Table 6). The results were supported by the findings of Shruthi and Salakinkop (2015).

\section{CONCLUSION}

In the laboratory experiment nano-encapsulated sulfentrazone herbicide was synthesized and characterized by using SEM and PSA which proved that the sulfentrazone was encapsulated perfectly and the size also reduced much compared to non-encapsulated (normal) sulfentrazone. In addition the zeta potential of encapsulated one is in correct range which made that powerful and stable. Then the synthesized encapsulated sulfentrazone was tested in the field experiment for its selectivity, efficacy and to optimize time and dosage of application. On the basis of both laboratory and field experiment (screening trial) it might be concluded that sulfentrazone @ 0.30 kg a.i. ha ${ }^{-1}$ with encapsulation and without encapsulation applied at 1 DBS and 2 DAS were produced lesser weed density and higher plant dry weight, seed yield. Even though both $\mathrm{T}_{1}$ and $\mathrm{T}_{5}$ are giving better results, $T_{1}$-sulfentrazone @ $0.30 \mathrm{~kg}$ a.i. ha ${ }^{-1}$ with encapsulation applied at 1 DBS is economically feasible to the farmer for getting higher gross returns, net returns and $B: C$ ratio. These better performing treatments in screening trial were compared with other herbicides and weeding methods in main field trial I and II. These trials revealed that sulfentrazone @ $0.30 \mathrm{~kg}$ a.i. ha ${ }^{-1}$ with encapsulation applied at 1 DBS was given lower weed density and higher seed yield, gross returns (92233 Rs ha-1), net returns (64,548 $\mathrm{Rs} \mathrm{ha}^{-1}$ ) and $\mathrm{B}: \mathrm{C}$ ratio (3.33). This concluded that sulfentrazone @ $0.30 \mathrm{~kg}$ a.i. ha ${ }^{-1}$ with encapsulation applied at 1 DBS is better alternative for the season long

weed management in blackgram without affecting the soil and ground water, as well as increasing the productivity.

\section{ACKNOWLEDGEMENT}

Authors are thankful to the Department of Agronomy and Department of Nano Science and Technology, TNAU, Coimbatore for rendering facilities whenever and wherever needed.

\section{REFERENCES}

Dayan, F.E., Armstrong, B.M., and Weete, J.D. (1998). Inhibitory activity of sulfentrazone and its metabolic derivatives on soybean (Glycine max) protoporphyrinogen oxidase. Journal of Agricultural and Food Chemistry. 46(5): 20242029.

Dayan, F. E., Green, H. M., Weete, J.D., and Hancock, H.G. (1996). Postemergence activity of sulfentrazone: effects of surfactants and leaf surfaces. Weed Science. 44(4): 797-803.

Duke, S.O., Dayan, F.E., Yamamoto, M., Duke, M.V.and Reddy, K.N. (1996). Protoporphyrinogen oxidase inhibitors-their current and future role. Procedings of International Weed Control Congress. 3: 775-780.

Gupta, V., Singh, M., Kumar, A., Sharma, B.C. and Kher, D. (2013). Influence of weed management practices on weed dynamics and yield of urdbean (Vigna mungo) under rainfed conditions of Jammu. Indian Journal of Agronomy. 58(2): 220-225.

Gustafson, D.I. (1989). Groundwater ubiquity score: a simple method for assessing pesticide leachability. Environmental Toxicology and Chemistry. An International Journal. 8(4): 339-357.

Hans, M.L. and Lowman, A.M. (2002). Biodegradable nanoparticles for drug delivery and targeting. Current Opinion in Solid State and Materials Science. 6(4): 319-327.

Krausz, R.F., Kapusta, G. and Matthews, J.L. (1998). Sulfentrazone for weed control in soybean (Glycine max). Weed Technology.12(4): 684-689. 
Martinez, C.O., Silva, C.M.M., Fay, E.F., Maia, A.D.H.N., Abakerli, R.B. and Durrant, L.R. (2008). Degradation of the herbicide sulfentrazone in a Brazilian Typic Hapludox soil. Soil Biology and Biochemistry. 40(4): 879-886.

Mohanraj, V.J., and Chen, Y. (2006). Nanoparticles-a review. Tropical Journal of Pharmaceutical Research. 5(1): 561-573.

Naidu, K.R.K., Ramana, A.V., Veeraraghavaiah, R. and Y. Ashoka Rani. (2011). Effect of pre and post emergence herbicides to the control of Vicia sativa in rice-fallow blackgram (Vigna mungo L.). The Andhra Agricultural Journal. 58(1): 5-8.
Reis, C.P., Neufeld, R.J., Ribeiro, A.J. and Veiga, F. (2006). Nanoencapsulation I. Methods for preparation of drugloaded polymeric nano particles. Nanomedicine: Nanotechnology, Biology and Medicine. 2(1). 8-2.

Shruthi, G.K. and Salakinkop, S.R. (2015). Efficacy of sequential application of pre and post-emergent herbicides in kharif green gram (Vigna radiata L.). Karnataka Journal of Agricultural Science. 28(2): 155-159.

Srivastava, T.K. (2003). Bio-efficacy of sulfentrazone against nutsedge (Cyperus rotundus) and other weeds in sugarcane. Indian Journal of Weed Science. 35(1 and 2): 82-86.

Walsh, K.D., Soltani, N., Hooker, D.C., Nurse, R.E. and Sikkema, P.H. (2015). Biologically effective rate of sulfentrazone applied pre-emergence in soybean. Canadian Journal of Plant Science. 95(2): 339-344. 